\title{
Bit Error-Rate Minimizing Detector for Amplify-and-Forward Relaying Systems Using Generalized Gaussian Kernel
}

\author{
Qasim Zeeshan Ahmed, Member, IEEE, Mohamed-Slim Alouini, Fellow IEEE, Sonia Aissa, Senior \\ Member, IEEE
}

\begin{abstract}
In this letter, a new detector is proposed for amplifyand-forward (AF) relaying system when communicating with the assistance of $L$ relays. The major goal of this detector is to improve the bit error rate (BER) performance of the receiver. The probability density function is estimated with the help of kernel density technique. A generalized Gaussian kernel is proposed. This new kernel provides more flexibility and encompasses gaussian and uniform kernels as special cases. The optimal window width of the kernel is calculated. Simulations results show that a gain of more than $1 \mathrm{~dB}$ can be achieved in terms of BER performance as compared to the minimum mean square error (MMSE) receiver when communicating over Rayleigh fading channels.
\end{abstract}

Index Terms-Cooperative Communications, Amplify-andForward (AF), Bit Error Rate, Kernel Density Estimation.

\section{INTRODUCTION}

Cooperative communication has received significant attention in the research community during the last decade. Amplify-and-forward (AF) relaying protocol has been the most popular due to its low complexity and similar bit error rate (BER) performance as compared to more complex protocols such as decode-and-forward (DF) [1,2]. Most of the existing work on AF-based relaying systems, with coherent detection, depends upon the assumption that perfect channel state information (CSI) is available at the relay and destination terminals [3]. The availability of CSI renders the noise to be Gaussian resulting in designing an optimal maximal likelihood (ML) detector for the AF relaying cooperative system [4]. However, in practical applications, this CSI can be acquired by using some estimation techniques $[5,6]$. The complexity of the system when measuring the channel estimates increases with the order of $O\left(L^{6.5}\right)$ where $L$ is the number of relays, which results in increased complexity [5].

In this paper, we propose a new detector for cooperative communication system when communicating with the assistance of AF protocol. An appropriate length of training sequence is used to estimate the probability density function (PDF) of the detected bit with the help of kernel density estimation [7], instead of measuring the CSI or assuming knowledge of the CSI. Furthermore, a generalized Gaussian kernel is proposed. This new kernel provides more flexibility, as it encompasses Gaussian, laplacian and uniform as special

The authors are with the Physical Sciences and Engineering Division, King Abdullah University of Science and Technology (KAUST), Thuwal, Makkah Province, Saudi Arabia. Email: \{qasim.ahmed, slim.alouini\} @kaust.edu.sa; sonia.aissa@ieee.org.

This work was supported by a KAUST Global Cooperative Research (GCR) fund. cases [8]. This kernel will assist us in measuring the distribution with heavier or lighter tails as compared to the normal Gaussian kernel. As the kernel width plays an important role to measure the PDF, the optimal kernel width is calculated. From our simulations, it is observed that this kernel is able to outperform the normal Gaussian kernel in terms of BER performance when optimal kernel width is selected.

\section{Cooperative System Model}

The basic cooperative communication system considered in this work is shown in Fig. 1. As illustrated, the source $(S)$ transmits data to the destination $(D)$ with the assistance of $L$ relays $\left(R_{1}, R_{2}, \cdots R_{L}\right)$. There exists no direct link between the source and the destination. The channel gains corresponding to the links from the source to the $l$ th relay and from the $l$ th relay to the destination are denoted by $h_{S R_{l}}$ and $h_{R_{l} D}$, respectively, and are assumed to be mutually independent. Any valid channel model distribution can be assumed. The data transmission takes place in two phases as shown in Fig. 1. Node $S$ transmits data to the relays in phase-I, while the data is amplified and forwarded to node $D$ through the relays in phase-II. Orthogonality is assumed in order to minimize interference between the relays, which can be achieved in the frequency or time domains [4].

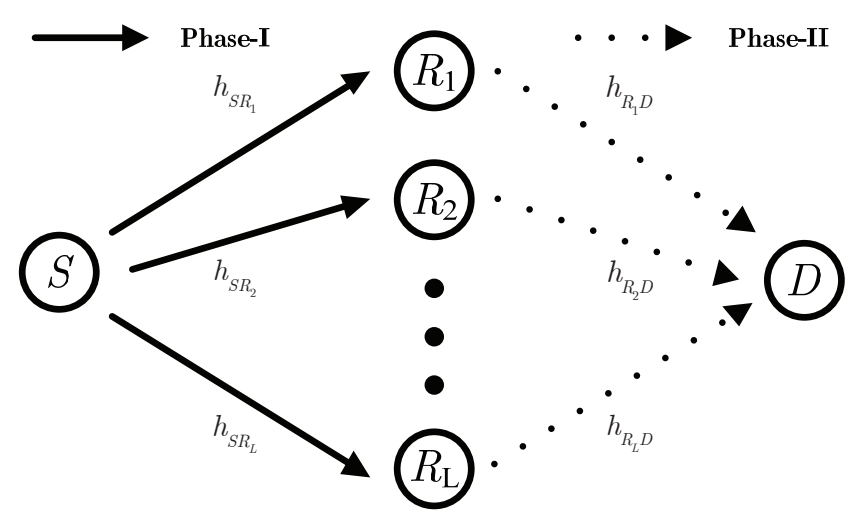

Figure 1. Schematic block diagram of a cooperative communication system when communicating with the assistance of $L$ AF-based relays.

\section{A. Phase-I: Transmission From Source}

Node $S$ broadcasts a binary phase shift keying (BPSK) ${ }^{1}$ signal $b$ with energy $E_{b}=1$ to all the relays, $R_{1}, R_{2}, \cdots R_{L}$,

\footnotetext{
${ }^{1}$ For complex modulation,e.g QPSK, QAM etc, $b=b_{R}+j b_{I}$, where $b_{R}$ is the real part and $b_{I}$ is the imaginary part of the signal.
} 
where $b=\{ \pm 1\}$ with equal probability. The received signals at the relays can be represented as

$$
y_{R_{l}}=h_{S R_{l}} b+n_{R_{l}}, \quad l=1,2, \cdots, L .
$$

where $n_{R_{l}}$ is additive white Gaussian noise (AWGN) with mean zero and variance $\sigma_{R_{l}}^{2} / 2$ per dimension.

\section{B. Phase-II: Transmission From Relays to Destination}

The received signal at the $l$-th relay is normalized by a factor $\zeta_{R_{l}}=\sqrt{\sigma_{S R_{l}}^{2}+\sigma_{R_{l}}^{2}}$. This ensures that the relay output satisfies an average or long-term power constraint of $E\left[\left|y_{R_{l}}\right|^{2}\right] / \zeta_{R_{l}}^{2}=1$, but allows the instantaneous transmit power to be much larger than the average $[6,9,10]$. This operation is performed at all the relays. Therefore, the signal received at node $D$ from the $l$-th relay is given by

$$
y_{l}=\underbrace{\frac{h_{R_{l} D} h_{S R_{l}}}{\zeta_{R_{l}}}}_{\text {signal-part }} b+\underbrace{\frac{h_{R_{l} D} n_{R_{l}}}{\zeta_{R_{l}}}+n_{D_{l}}}_{\text {noise-part }}, \quad l=1,2, \cdots, L(2)
$$

From the received signal $y_{l}$, it can be observed that the noisepart is not Gaussian, as the $n_{R_{l}}$ gets amplified and forwarded over the relay-destination channel. A different noise power will be encountered at node $D$ due to different relay-destination channels.

\section{Receiver Structure}

As the desired signal $b$ arrives at the destination with the assistance of $L$ relays, $L$ copies of this signal need to be collected. The vector form of the received signal can be represented as

$$
\boldsymbol{y}=\boldsymbol{h} b+\boldsymbol{n},
$$

where $\boldsymbol{h}$ is defined as

$$
\boldsymbol{h}=\left[\frac{h_{R_{1} D} h_{S R_{1}}}{\zeta_{R_{1}}}, \frac{h_{R_{2} D} h_{S R_{2}}}{\zeta_{R_{2}}}, \cdots, \frac{h_{R_{L} D} h_{S R_{L}}}{\zeta_{R_{L}}}\right]^{T}
$$

and $n$ can be represented as

$$
\begin{aligned}
\boldsymbol{n} & =\left[\frac{h_{R_{1} D} n_{R_{1}}}{\zeta_{R_{1}}}+n_{D_{1}}, \cdots, \frac{h_{R_{L} D} n_{R_{L}}}{\zeta_{R_{L}}}+n_{D_{L}}\right]^{T} \\
& =\left[n_{1}, \cdots, n_{L}\right]^{T}
\end{aligned}
$$

where $n_{1}, n_{2} \cdots, n_{L}$ are the noise-part of the received signal through relays $R_{1}, R_{2}, \cdots, R_{L}$, as represented in (2).

\section{Detection FOR COOPERATIVE COMMUNICATION SYSTEMS}

For a low-complexity system, linear filtering is preferred and the receiver filter can be characterized as

$$
z=\boldsymbol{w}^{H} \boldsymbol{y}=\boldsymbol{w}^{H} \boldsymbol{h} b+\boldsymbol{w}^{H} \boldsymbol{n},
$$

where $\boldsymbol{w}=\left[w_{1}, \cdots, w_{L}\right]^{T}$ and $w_{l}$ is the $l$-th tap complex valued filter coefficient. As our signal $b$ is BPSK modulated, then the real part of $z_{R}=\Re(z)$ will be of interest. ${ }^{2}$ The estimate of the desired bit $b$ is expressed as

$$
\hat{b}=\operatorname{sgn}\left(z_{R}\right)=\operatorname{sgn}\left(\Re\left(\boldsymbol{w}^{H} \boldsymbol{h} b\right)+\Re\left(\boldsymbol{w}^{H} \boldsymbol{n}\right)\right),
$$

where $\operatorname{sgn}(\cdot)$ is the sign function. ${ }^{3}$ From Eq. (7), it can be observed that in order to detect the desired bit we need an optimal weight vector $\boldsymbol{w}$. This optimal weight vector $\boldsymbol{w}$ can depend upon different criteria. Usually, this $\boldsymbol{w}$ is measured with the help of minimum mean square error (MMSE) criterion [11]. However, improved reliability can be achieved if we could minimize the probability of error instead of minimizing the MSE [12]. Therefore, for equally likely $b= \pm 1$, the probability of error ${ }^{4}$ is given by

$$
P_{e}(\boldsymbol{w})=\int_{-\infty}^{0} p\left(z_{R}, \boldsymbol{w}\right) d z_{R}
$$

The PDF of $p\left(z_{R}, \boldsymbol{w}\right)$ is difficult to determine since the noisepart and the signal-part are related with one another and the noise-part is not Gaussian. A reliable estimate of this PDF could be produced with the help of kernel density estimation [7]. Given a training length $T L$, the estimate of the PDF can be computed with the kernel $K(\cdot)$ as

$$
\hat{p}_{z_{R}}\left(z_{R}, \boldsymbol{w}\right)=\frac{1}{T L} \sum_{i=1}^{T L} \frac{1}{\rho} K\left(\frac{z_{R}-z_{R}(i)}{\rho}\right),
$$

where $\rho$ is the window width. ${ }^{5}$ Usually, Gaussian kernel is employed due to its simplicity. However, this kernel is not appropriate for heavier and lighter tailed distributions [7].

A generalized Gaussian kernel is proposed where the shape of kernel is measured with the help of a shape parameter $\beta$. This parameter will assist us in estimating heavier-tailed distributions for $\beta<2$, lighter distributions for $\beta>2$ and will be equal to the Gaussian kernel for $\beta=2$. With the help of the generalized Gaussian kernel, the estimated PDF can be expressed as

$$
\begin{aligned}
\hat{p}_{z_{R}}\left(z_{R}, \boldsymbol{w}\right) & =\frac{1}{T L} \sum_{i=1}^{T L} \frac{1}{\rho} \frac{\beta}{2 \alpha \Gamma(1 / \beta)} \\
& \times \exp \left(-\left(\frac{\left|z_{R}-\Re\left(\boldsymbol{w}^{H}(i) \boldsymbol{y}(i)\right)\right|}{\alpha \rho}\right)^{\beta}\right),
\end{aligned}
$$

where $\alpha^{2}=\sigma^{2} \Gamma(1 / \beta) / \Gamma(3 / \beta)$ [8]. Substituting (10) in (8), the approximated probability of error, $\hat{P}_{e}(w)$, can be written as

$$
\begin{aligned}
\hat{P}_{e}(\boldsymbol{w}) & =\int_{-\infty}^{0} \hat{p}\left(z_{R}, \boldsymbol{w}\right) d z_{R} \\
& =\frac{1}{T L} \sum_{i=1}^{T L}\left[\frac{1}{2}+\frac{\operatorname{sgn}\left(-\Re\left(\boldsymbol{w}^{H}(i) \boldsymbol{y}(i)\right)\right)}{2 \Gamma(1 / \beta)}\right. \\
& \left.\times \gamma\left(\frac{1}{\beta},\left(\frac{\mid \Re\left(\boldsymbol{w}^{H}(i) \boldsymbol{y}(i) \mid\right.}{\alpha \rho}\right)\right)\right],
\end{aligned}
$$

${ }^{2}$ For complex modulation, the imaginary part of $z$ will be calculated as $z_{I}=\Im(z)$.

${ }^{3}$ For complex modulation, $\hat{b}=\operatorname{sgn}\left(z_{R}\right)+j \operatorname{sgn}\left(z_{I}\right)$.

${ }^{4}$ For complex modulation, $P_{e}(\boldsymbol{w})=\int_{-\infty}^{0} p\left(z_{R}, \boldsymbol{w}\right) d z_{R}+p\left(z_{I}, \boldsymbol{w}\right) d z_{I}$. ${ }^{5} \hat{p}_{z_{I}}\left(z_{I}, \boldsymbol{w}\right)$ can be simplify measured by using $z_{I}(i)$ instead of $z_{R}(i)$. 
where $\gamma(s, x)=\int_{0}^{x} t^{s-1} \exp (-t) d t$ is the lower incomplete Gamma function [13].

In order to minimize (11), the derivative of $\hat{P}_{e}(\boldsymbol{w})$ is calculated:

$$
\begin{aligned}
\nabla \hat{P}_{e}(\boldsymbol{w}) & =\frac{1}{T L} \sum_{i=1}^{T L}\left[-\frac{\beta \operatorname{sgn}(b(i))}{4 \alpha \rho \Gamma(1 / \beta)}\right. \\
& \left.\times \exp \left(-\left(\frac{\left|\Re\left(\boldsymbol{w}^{H}(i) \boldsymbol{y}(i)\right)\right|}{\alpha \rho}\right)^{\beta}\right) \boldsymbol{y}(i)\right] .
\end{aligned}
$$

Finally, the weight vector $\boldsymbol{w}$ can be computed with the assistance of steepest-descent algorithms, which can be represented as

$$
\boldsymbol{w}(i+1)=\boldsymbol{w}(i)-\mu \nabla \hat{P}_{e}(\boldsymbol{w}(i)) .
$$

The proposed detector will now operate in two modes, the training mode and the decision directed (DD) mode. In the training mode, the weight vector updating can be formulated as

$$
\begin{aligned}
\boldsymbol{w}(i+1) & =\boldsymbol{w}(i)+\mu \frac{\beta \operatorname{sgn}(b(i))}{4 \alpha \rho \Gamma(1 / \beta)} \\
& \times \exp \left(-\left(\frac{\left|\Re\left(\boldsymbol{w}^{H}(i) \boldsymbol{y}(i)\right)\right|}{\alpha \rho}\right)\right) \boldsymbol{y}(i)
\end{aligned}
$$

where the step-size $\mu, \rho$ and $\beta$ are required to be adjusted to minimize the BER. Usually, smaller $\mu$ leads to slower convergence but better BER performance. Parameter $\beta$ can be calculated with the help of kurtosis $\kappa$ which is given by [8]:

$$
\kappa=\frac{\Gamma(5 / \beta) \Gamma(1 / \beta)}{\Gamma^{2}(3 / \beta)} .
$$

For a known $\beta$, the optimal $\rho$ can be adjusted as shown in the appendix. After completing the training mode, the detector is switched to the DD-mode, where the weight vector updating is done according to

$$
\begin{aligned}
\boldsymbol{w}(i+1) & =\boldsymbol{w}(i)+\mu \frac{\beta \operatorname{sgn}(\hat{b}(i))}{4 \alpha \rho \Gamma(1 / \beta)} \\
& \times \exp \left(-\left(\frac{\left|\Re\left(\boldsymbol{w}^{H}(i) \boldsymbol{y}(i)\right)\right|}{\alpha \rho}\right)\right) \boldsymbol{y}(i),(16)
\end{aligned}
$$

where $\hat{b}(i)$ is the estimate of $b(i)$. Next, we provide simulation results sustaining the above analysis.

\section{Simulation Results ANd Discussion}

In this section, the BER performance of the proposed cooperative communication system with $L$ relays is investigated. In our simulations, the channel gains were assumed to obey the Rayleigh distribution. Furthermore, the normalized channel Doppler frequency was fixed to $1 \times 10^{-5}$. The training length is set to 200 , the frame length is fixed to 1000 and the step-size is set to $\mu=0.25$. Further, it is assumed that the transmitted signal has unit power and that the destination and all the relays have the same noise power.

Fig. 2 illustrates the learning performance of the cooperative communication system carried out with the assistance of

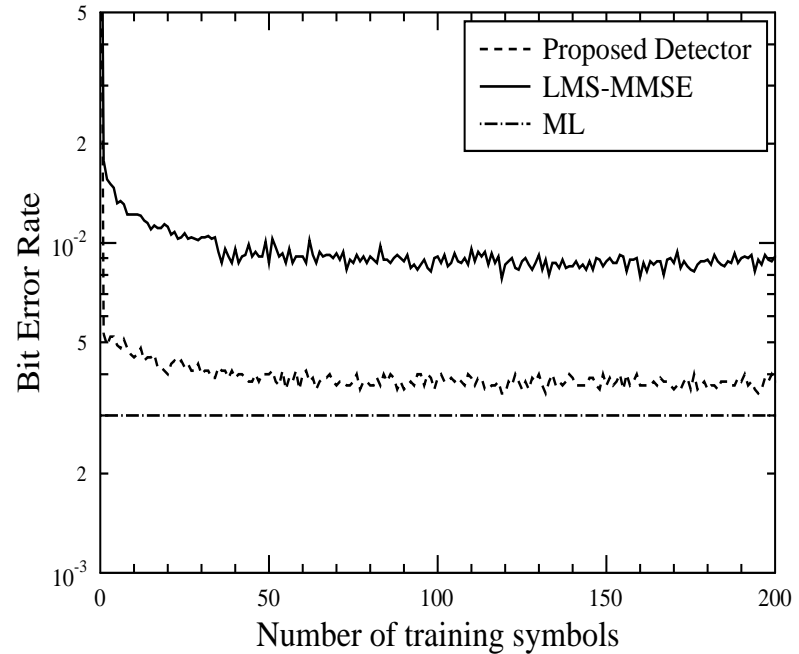

Figure 2. Learning curves of the cooperative communication system with $L=2$ available relays.

$L=2$ relays. In our simulations, the BER performance was obtained by averaging over 100,000 independent realizations of the channel at $E_{b} / N_{0}=14 \mathrm{~dB}$. Based on the results, it can be observed that a training length of 200 bits is sufficient for convergence of the algorithm. The proposed detector is able to approach maximum likelihood (ML) detector in terms of BER. It can be observed that the BER convergence of the proposed detector is superior to the MMSE detector which is implemented with the help of least mean square (LMS).

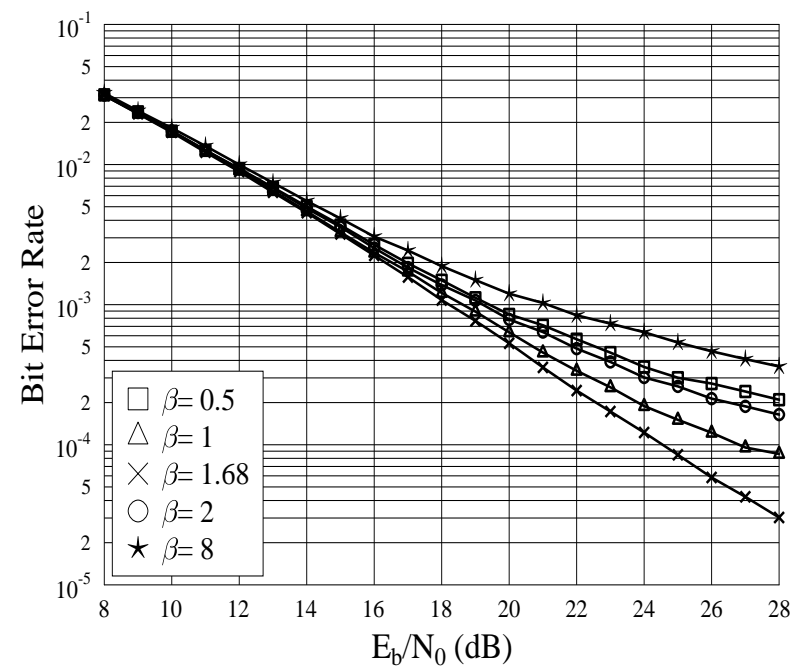

Figure 3. BER performance of an AF cooperative communication system with $L=2$ relays.

Fig. 3 shows the effect of different values of the shape parameter $\beta$ for the $\mathrm{AF}$ relaying system when the communication is carried over Rayleigh fading channels. From the figure, it can be observed that generalized Gaussian density with $\beta=1.68$ yields much better BER performance as compared to $\beta=1$ Laplacian density, $\beta=2$ Gaussian density and $\beta=8$ approximate uniform density. Furthermore, from the plots it 
can be observed that approximating the noise as Gaussian is not appropriate. Moreover, a significant gain can be observed by approximating the noise as a generalized Gaussian with an appropriate shape parameter $\beta$.

Fig. 4 shows the BER performance of the cooperative communication system, as a function of the SNR per bit, when communicating over Rayleigh fading channels. It can be observed that higher order diversity can be obtained as the number of available relays increases. Furthermore, it can be observed that a BER gain of more than $1 \mathrm{~dB}$ can be obtained by employing the proposed detector as compared to the conventional relay-assisted MMSE detector. The value of $\beta$ was determined with the help of (15). The proposed detector is able to perform close to the ML-detector.

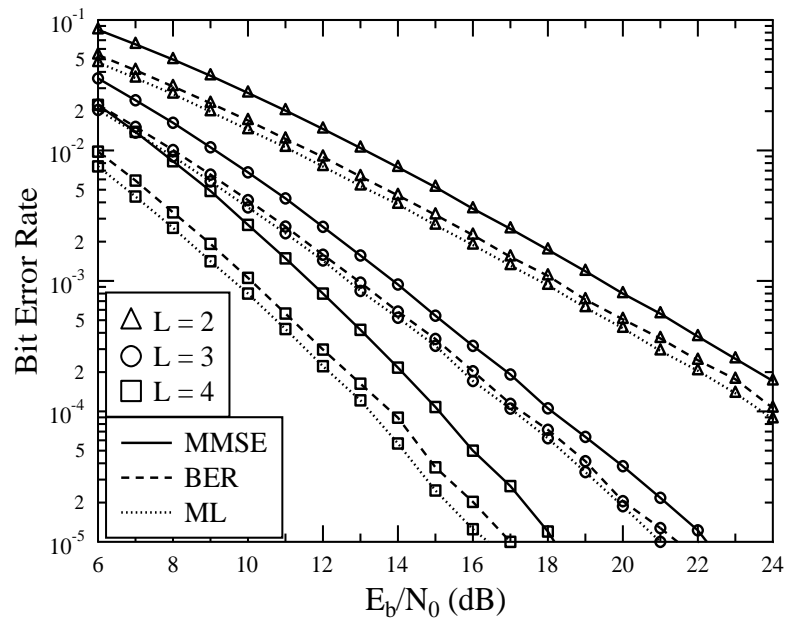

Figure 4. BER performance of a cooperative communication system when communicating with the assistance of $L$ relays.

\section{Conclusions}

This paper proposed a new detector for amplify-and-forward relaying systems. This detector has the capability of improving the bit error rate performance when communicating over Rayleigh fading channels. The detector is independent from channel estimation. A training sequence is used to measure the probability density function of the detected signal with the help of kernel density estimation. A new generalized Gaussian kernel, which has more flexibility as compared to the normal kernel, was proposed. The optimal kernel width was calculated. Simulation results we presented and attest that a gain of more than $1 \mathrm{~dB}$ is possible as compared to the conventional MMSE detection schemes.

\section{APPENDIX}

The kernel width, $\rho$, plays an important role in the PDF estimation. A smaller value of $\rho$ will make the density spiky and hard to interpret, while a larger value of $\rho$ will oversmooth the density [7]. Therefore, an optimized value of $\rho$ will be required so that the mean integrate error between the estimated density and the true one can be minimized [7]. The optimized value of $\rho$ is given by [7]:

$$
\rho=\left(\frac{\int(K(t))^{2} d t}{(T L) \int\left(\frac{d^{2} P_{e}\left(z_{R}, \boldsymbol{w}\right)}{d z_{R}^{2}}\right) d z_{R}}\right)^{\frac{1}{5}},
$$

where $(K(t))^{2}$ is the square of the kernel density. For a generalized Gaussian kernel, the integral of the latter is found to be equal to

$$
\int(K(t))^{2} d t=2^{(-1-1 / \beta)} \beta \sqrt{\frac{\Gamma(3 / \beta)}{\Gamma^{2}(1 / \beta)}},
$$

and we have

$$
\begin{aligned}
\int \frac{d^{2} P_{e}\left(z_{R}, \boldsymbol{w}\right)}{d z_{R}^{2}} d z_{R} & =2^{(-5+3 / \beta)} \\
& \times \frac{\beta^{3}(\beta-1)(2 \beta-1) \Gamma(2-3 / \beta)}{\left(\alpha^{5} \Gamma^{2}(1 / \beta)\right)},
\end{aligned}
$$

for $\beta>1.5$. Substituting (18) and (19) in (17), we get

$$
\left.\rho=\left(\frac{\alpha^{5} \sqrt{\Gamma(1 / \beta) \Gamma(3 / \beta)}}{(T L) 2^{(4 / \beta-4)} \beta^{2}(\beta-1)(2 \beta-1) \Gamma(2-3 / \beta)}\right)\right)^{\frac{1}{5}} .
$$

Then, plugging the value of $\alpha$ in the above equation yields

$$
\rho=\left(\frac{\sigma^{5} \Gamma^{3}(1 / \beta) / \Gamma^{2}(3 / \beta)}{(T L) 2^{(4 / \beta-4)} \beta^{2}(\beta-1)(2 \beta-1) \Gamma(2-3 / \beta)}\right)^{\frac{1}{5}} \text {. }
$$

In particular, making $\beta=2.0$ in the above, the equation reduces to $\rho=1.06 \sigma(T L)^{-1 / 5}$, which is equivalent to the proposed optimal window for the Gaussian kernel [7].

\section{REFERENCES}

[1] J. N. Laneman, D. N. C. Tse, and G. W. Wornell, "Cooperative diversity in wireless networks: Efficient protocols and outage behavior,"IEEE Trans. Inform. Theory, vol. 50, no. 12, pp. 3062-3080, Dec. 2004.

[2] Y. Zhao, R. Adve, and T. J. Lim, "Improving amplify-and-forward relay networks: Optimal power allocation versus selection," IEEE Trans. Wireless Commun., vol. 6, no. 8, pp. 3114-3122, Aug. 2007.

[3] H. Mheidat and M. Uysal, "Non-coherent and mismatched-coherent receivers for distributed STBCs with amplify-and-forward relaying,"IEEE Trans. Wireless Commun., vol. 6, no. 11, pp. 4060-4070, Nov. 2007.

[4] D. Chen and J. N. Laneman, "Modulation and demodulation for cooperative diversity in wireless systems,"IEEE Trans. Wireless Commun., vol. 5, no. 7, pp. 1785-1794, Jul. 2006.

[5] F. Gao, T. Cui, and A. Nallanathan, "On channel estimation and optimal training design for amplify and forward relay networks,"IEEE Trans. Wireless Commun., vol. 7, no. 5, pp. 1907-1916, May 2008.

[6] C. S. Patel and G. L. Stuber, "Channel estimation for amplify and forward relay based cooperation diversity systems,"IEEE Trans. Wireless Commun., vol. 6, no. 6, pp. 2348-2355, Jun. 2007.

[7] B. W. Silverman, Density Estimation. Chapman Hall, London, 1996.

[8] M. Novey, T. Adali, and A. Roy, "A complex generalized Gaussian distribution- characterization, generation and estimation," IEEE Trans. Signal Processing, vol. 58, no. 3, pp. 1427-1433, Mar. 2010.

[9] Y. Zhu, P-Y. Kam, and Y. Xin, "Non-Coherent Detection form Amplifyand-Forward Relay Systems in a Rayleigh Fading Environment,"IEEE Global Communications Conference (GLOBECOM'07), Washington, DC, USA, pp. 1658-1662, Nov. 2007.

[10] D. Chen, and J. N. Laneman, "Cooperative diversity for wireless fading channels without channel state information,"IEEE 38th Asilomar Conference on Signals, Systems and Computers (ACSSC'04), Pacific Grove, California, USA, pp. 1307-1312, Nov. 2004.

[11] S. Haykin, Adaptive Filter Theory. Prentice Hall, 4th ed., 2002.

[12] S. Chen, S. Tan, L. Xu, and L. Hanzo, "Adaptive minimum error-rate filtering design: A Review," Elsevier Signal Processing, vol. 88, no. 7, pp. 1671-1679, 2008.

[13] M. K. Simon, Probability Distributions Involving Gaussian Random Variables: A Handbook for Engineers, Scientists and Mathematicians. Springer, 2006. 